\title{
A DFT Study on the Mechanism of Phosphodiester Cleavage Mediated by Monozinc Complexes
}

\author{
Yubo Fan and Yi Qin Gao* \\ Department of Chemistry, Texas A \& M University, College Station, TX, 77843-3255 \\ E-mail: $\underline{\text { yiqin@mail.chem.tamu.edu }}$
}

Complete description for Reference (26):

Gaussian 03, Revision B.05 and C.02 Frisch, M. J.; Trucks, G. W.; Schlegel, H. B.; Scuseria, G. E.; Robb, M. A.; Cheeseman, J. R.; Montgomery, Jr., J. A.; Vreven, T.; Kudin, K. N.; Burant, J. C.; Millam, J. M.; Iyengar, S. S.; Tomasi, J.; Barone, V.; Mennucci, B.; Cossi, M.; Scalmani, G.; Rega, N.; Petersson, G. A.; Nakatsuji, H.; Hada, M.; Ehara, M.; Toyota, K.; Fukuda, R.; Hasegawa, J.; Ishida, M.; Nakajima, T.; Honda, Y.; Kitao, O.; Nakai, H.; Klene, M.; Li, X.; Knox, J. E.; Hratchian, H. P.; Cross, J. B.; Bakken, V.; Adamo, C.; Jaramillo, J.; Gomperts, R.; Stratmann, R. E.; Yazyev, O.; Austin, A. J.; Cammi, R.; Pomelli, C.; Ochterski, J. W.; Ayala, P. Y.; Morokuma, K.; Voth, G. A.; Salvador, P.; Dannenberg, J. J.; Zakrzewski, V. G.; Dapprich, S.; Daniels, A. D.; Strain, M. C.; Farkas, O.; Malick, D. K.; Rabuck, A. D.; Raghavachari, K.; Foresman, J. B.; Ortiz, J. V.; Cui, Q.; Baboul, A. G.; Clifford, S.; Cioslowski, J.; Stefanov, B. B.; Liu, G.; Liashenko, A.; Piskorz, P.; Komaromi, I.; Martin, R. L.; Fox, D. J.; Keith, T.; Al-Laham, M. A.; Peng, C. Y.; Nanayakkara, A.; Challacombe, M.; Gill, P. M. W.; Johnson, B.; Chen, W.; Wong, M. W.; Gonzalez, C.; and Pople, J. A.; Gaussian, Inc., Wallingford CT, 2004.

Cartesian coordinates (Aryl and alkyl hydrogen atoms are not included) for all species shown in Figure 1 and 2 are provided below: 
Supporting Information

1

\begin{tabular}{|c|c|c|c|}
\hline $\mathrm{Zn}$ & -8.000 & 8.000 & 0.000 \\
\hline $\mathrm{N}$ & -8.051 & 6.893 & -1.957 \\
\hline $\mathrm{C}$ & -6.766 & 7.195 & -2.634 \\
\hline $\mathrm{C}$ & -9.213 & 7.450 & -2.697 \\
\hline C & -8.234 & 5.442 & -1.696 \\
\hline $\mathrm{C}$ & -4.376 & 6.558 & -2.029 \\
\hline $\mathrm{C}$ & -4.702 & 8.024 & 0.308 \\
\hline $\mathrm{C}$ & -5.581 & 7.166 & -1.681 \\
\hline $\mathrm{C}$ & -3.294 & 6.673 & -1.132 \\
\hline $\mathrm{C}$ & -3.446 & 7.411 & 0.035 \\
\hline $\mathrm{N}$ & -5.764 & 7.851 & -0.525 \\
\hline C & -7.048 & 3.690 & -0.295 \\
\hline $\mathrm{C}$ & -7.231 & 5.437 & 1.868 \\
\hline $\mathrm{C}$ & -7.597 & 4.969 & -0.399 \\
\hline $\mathrm{C}$ & -6.570 & 3.278 & 0.968 \\
\hline C & -6.661 & 4.140 & 2.052 \\
\hline $\mathrm{N}$ & -7.685 & 5.832 & 0.640 \\
\hline $\mathrm{C}$ & -11.727 & 7.215 & -2.290 \\
\hline $\mathrm{C}$ & -11.247 & 8.027 & 0.328 \\
\hline $\mathrm{C}$ & -10.446 & 7.488 & -1.805 \\
\hline $\mathrm{C}$ & -12.810 & 7.346 & -1.395 \\
\hline $\mathrm{C}$ & -12.582 & 7.755 & -0.087 \\
\hline $\mathrm{N}$ & -10.203 & 7.851 & -0.525 \\
\hline $\mathrm{N}$ & -4.905 & 8.790 & 1.425 \\
\hline $\mathrm{H}$ & -5.619 & 9.532 & 1.333 \\
\hline $\mathrm{H}$ & -4.063 & 9.090 & 1.908 \\
\hline $\mathrm{N}$ & -10.944 & 8.533 & 1.567 \\
\hline $\mathrm{H}$ & -9.913 & 8.524 & 1.839 \\
\hline $\mathrm{H}$ & -11.636 & 8.399 & 2.297 \\
\hline $\mathrm{N}$ & -7.379 & 6.311 & 2.895 \\
\hline $\mathrm{H}$ & -6.888 & 6.116 & 3.758 \\
\hline $\mathrm{H}$ & -7.659 & 7.304 & 2.666 \\
\hline 0 & -8.276 & 8.590 & 1.861 \\
\hline $\mathrm{H}$ & -8.019 & 9.526 & 1.958 \\
\hline P & -7.339 & 11.087 & -0.537 \\
\hline 0 & -6.557 & 11.056 & 0.786 \\
\hline 0 & -8.052 & 9.827 & -1.085 \\
\hline 0 & -8.350 & 12.403 & -0.631 \\
\hline 0 & -6.322 & 11.486 & -1.870 \\
\hline $\mathrm{C}$ & -5.511 & 12.586 & -1.934 \\
\hline $\mathrm{C}$ & -5.062 & 12.938 & -3.227 \\
\hline $\mathrm{C}$ & -4.211 & 14.028 & -3.404 \\
\hline $\mathrm{C}$ & -3.811 & 14.769 & -2.279 \\
\hline $\mathrm{C}$ & -4.245 & 14.430 & -0.988 \\
\hline $\mathrm{C}$ & -5.095 & 13.335 & -0.810 \\
\hline $\mathrm{N}$ & -2.916 & 15.920 & -2.458 \\
\hline 0 & -2.577 & 16.561 & -1.447 \\
\hline 0 & -2.542 & 16.193 & -3.613 \\
\hline C & -8.782 & 13.122 & 0.554 \\
\hline $\mathrm{C}$ & -10.101 & 12.568 & 1.103 \\
\hline $\mathrm{C}$ & -10.758 & 13.565 & 2.073 \\
\hline 0 & -9.801 & 11.339 & 1.790 \\
\hline $\mathrm{H}$ & -10.405 & 10.627 & 1.499 \\
\hline
\end{tabular}

TS-2

$\mathrm{Zn}$

$\mathrm{N}$

C

C

C

C

C

C
C

C

$\mathrm{N}$

C

C

C

C

N

C

C

C

C

$\mathrm{N}$

N

$\mathrm{H}$

$\mathrm{H}$

$\mathrm{N}$
$\mathrm{H}$

$\mathrm{H}$

$\mathrm{H}$
$\mathrm{N}$

$\mathrm{N}$
$\mathrm{H}$

$\mathrm{H}$

$\mathrm{O}$

$\mathrm{H}$

P

0

0

0

C

C

C

C

C

8.000
7.971
9.229
6.760
7.868

11.671

11.277

10.425

12.764

12.577

10.211

9.221

8.940

8.570

9.755

9.612

8.435

4.294

4.792

5.564

3. 238

3.476

5.825

11.047

10.284

11.882

5.111

6.752

4.306

8.740

9.296

8.428

7.753

8.138

8.379

8.962

7.961

7.843

9.920

10.872

12.066

13.120

12.994

11.820

10.764

14.099

13.962

15.132

6.424

5.764

5.903

6.432

5.720
8.000

$$
6.815
$$

7.181

7.279

5.362

6.745

8.141

7.235

6.950

7.654

7.878

3.711

5.404

4. 942

3. 319

4.157

5.790

6.888

8.171

7. 321

7.059

7.705

7.878

8.861

9.567

9. 198

8.951

8.689

9.111

6.232

6.074

7. 195

8.599

9.552

11.049

10.780

9.850

12.571

11.383

12.257

12.250

13.105

13.987

14.016

13.159

14.881

15.653

14.827

12.834

12.241

13.140

11.003

10.000
0.000

$-1.856$

$-2.553$

$-2.584$

$-1.562$

$-2.005$

0.368

$-1.614$

$-1.136$

0.045

$-0.439$

$-0.207$

1.974

$-0.283$

1.038

2.133

0.766

$-2.026$

0.406

$-1.646$

$-1.101$

0.106

$-0.439$

1.511

1. 474

1.978

1. 488

2.060

2.095

3.039

3.870

2.846

2.008

1.969

$-0.017$

1. 419

$-0.934$

$-0.458$

$-0.867$

$-0.488$

$-1.260$

$-0.954$

0.136

0.909

0.602

0.469

1.438

$-0.231$

$-0.514$

0.743

1.984

0.919

1. 178 


\section{Supporting Information}

$3 a$

$\mathrm{Zn}$

$\mathrm{N}$

C

C

C

C

C

$\mathrm{N}$

C

C

C

C

C

$\mathrm{N}$

$\mathrm{N}$

$\mathrm{H}$

$\mathrm{H}$

H

$\mathrm{N}$

O

$\mathrm{H}$

C

C

C $\quad-9.965$

C $\quad-9.630$

O $\quad-9.522$

$\mathrm{H} \quad-10.472$
$-8.000$

$-9.212$

$-8.837$

$-8.764$

$-10.668$

$-9.222$

$-7.833$

$-8.768$

$-8.985$

$-8.283$

$-8.133$

$-12.310$

$-10.629$

$-11.087$

$-12.699$

$-11.867$

$-10.246$

$-9.223$

$-7.853$

$-8.736$

$-9.057$

$-8.356$

$-8.133$

$-7.116$

$-6.428$

$-6.750$

$-7.039$

$-7.387$

$-6.800$

$-9.807$

$-9.950$

$-8.846$

$-7.461$

$-6.465$

$-4.972$

$-5.314$

$-6.182$

$-3.366$

$-4.808$

$-4.022$

$-4.087$

$-3.328$

$-2.478$

$-2.389$

$-3.150$

$-1.688$

$-0.941$

$-1.796$

$-2.780$

$-3.533$

$-2.973$

$-4.875$

$-6.175$
0.000

$-1.837$

$-2.550$

$-2.567$

$-1.547$

$-2.039$

0.340

$-1.627$

$-1.190$

$-0.008$

$-0.447$

$-0.220$

1. 971

$-0.281$

1.016

2.117

0.774

$-2.001$

0.367

$-1.635$

$-1.090$

0.094

$-0.447$

1.483

1. 462

1. 931

1. 458

2.130

2.008

3.043

3.857

2. 847

2. 013

1.950

0.046

1. 487

$-0.909$

$-0.443$

$-0.756$

$-0.376$

$-1.162$

$-0.843$

0.281

1.072

0.750

0.629

1. 628

$-0.085$

$-0.268$

0.882

2. 275

0.709

1. 179

TS-4a

$\mathrm{Zn}$

$\mathrm{N}$

C

C

C

C

C

C

C

C

N

C

C

C

C

C

N

C

C

C

C

C

N

$\mathrm{N}$
$\mathrm{H}$

$\mathrm{H}$
$\mathrm{H}$

N

$\mathrm{H}$

$\mathrm{H}$

$\mathrm{N}$

$\mathrm{H}$

$\mathrm{H}$

O

P

$\mathrm{P}$

0

o

0

$\mathrm{O}$

C

C

C

C

C

$\mathrm{N}$

O

C

c

C

$\mathrm{O}$

8.000
8.037
9.290
6.821
7.976
11.711
11.340
10.480
12.790
12.615
10.276
8.956

8.233

8.407

9.156

8.777

8.094

4.362

4. 822

5.621

3. 301

3. 518

5.859

11.094

10.537

11.936

5.119

8.344

4. 319

7.771

8.071

7.649

8.358

9.335

8.436

9.408

8.001

7.835

9.744

10.709

11.604

12.657

12.841

11.969

10.914

13.946

14.087

14.707

6.573

6.355

7.001

6.896

5.849

$-8.000$

$$
-9.150
$$

$-8.732$

$-8.660$

$-10.614$

$-9.324$

$-7.981$

$-8.787$

$-9.210$

$-8.523$

$-8.186$

$-12.336$

$-10.714$

$-11.065$

$-12.802$

$-12.004$

$-10.237$

$-9.145$

$-7.978$

$-8.694$

$-9.044$

$-8.453$

$-8.186$

$-7.214$

$-6.326$

$-7.009$

$-7.309$

$-6.418$

$-7.033$

$-9.937$

$-10.185$

$-8.934$

$-7.423$ 
Supporting Information

$3 b$

$\mathrm{Zn}$

C

c

C

C

C

C

$\mathrm{N}$

C

C

$\mathrm{N}$

$-5.474$

$-4.008$

$-10.888$

$-7.469$

$-11.695$

$-8.419$

$-8.164$

$-8.528$

$-7.668$

$-6.754$

$-7.633$

$-6.819$

$-7.973$

$-8.062$

$-6.111$

$-5.131$

$-4.032$

$-2.946$

$-2.932$

$-4.004$

$-5.092$

$-1.794$

$-1.807$

$-0.851$

$-9.384$

$-9.833$

$-9.387$

$-9.276$

$-10.188$
8.000

6.837

7.267

7. 318

5.370

6.681

8.032

7.214

6.802

7.492

7.817

3.647

5.290

4.922

3.191

3.999

5.758

6.803

8.015

7.283

6.901

7.514

7.817

8.792

9.630

9.041

8.701

9.600

8.971

6.081

5.846

7.082

8.557

8.245

11.021

10.777

9.770

12.592

11.332

12.199

12.195

13.046

13.929

13.958

13.106

14.815

15.590

14.759

13.035

12.201

12.791

10.917

9.463
0.000

$-1.892$

$-2.571$

$-2.595$

$-1.666$

$-2.028$

0.365

$-1.634$

$-1.142$

0.054

$-0.437$

$-0.052$

2.030

$-0.257$

1.274

2.322

0.752

$-2.048$

0.416

$-1.660$

$-1.120$

0.111

$-0.437$

1.504

1.342

2.001

1.568

1.864

2.123

3.027

3.979

2. 827

2.015

2.199

$-0.048$

1.309

$-0.960$

$-0.502$

$-1.059$

$-0.808$

$-1.719$

$-1.545$

$-0.447$

0.466

0.291

$-0.250$

0.730

$-1.070$

$-0.137$

1.080

2.430

0.828

1.445
TS $-4 \mathrm{~b}$

$\mathrm{Zn}$

8.000
8.051

8.000

0.000

$\mathrm{N}$

9.302

6.836

8.000

11.718

11.388

10.498

12.809

12.655

10.311

8.864

8.083

8.353

8.990

8.584

8.012

4.381

4.817

5.631

3. 317

3.522

5.858

11.181

10.555

12.039

5.097

8.574

4.287

7.595

7.843

7.514

8.313

9.210

8.336

9.118

8.041

7.939

9.897

10.862

11.983

13.057

13.033

11.936

10.861

14.157

14.109

15.121

6.612

6.125

6.533

6.685

5.805

$\mathrm{O}$
$\mathrm{H}$
6.846

7.268

7.331

5.376

6.651

8.032

7.207

6.765

7.468

7.826

3.639

5.304

4.922

3.186

4.005

5.764

6.818

8.013

7.297

6.909

7.514

7.826

8.804

9.624

9.065

8.689

9.707

8.961

6.116

5.882

7.120

8.582

8.250

11.030

10.770

9.774

12.611

12.222

12.239

13.096

13.966

13.976

13.116

14.861

15.624

14.822

13.062

12.211

12.781

10.928

9.440
11.346

$-1.904$

$-2.583$

$-2.613$

$-1.685$

$-2.040$

0.339

$-1.651$

$-1.157$

0.033

$-0.460$

$-0.065$

2.012

$-0.275$

1.263

2.307 


\section{Supporting Information}

$3 c$

$\mathrm{Zn}$

$\mathrm{N}$

C

C

C

C

C

C

C

C

C

C

C

C

$\mathrm{N}$

$\mathrm{N}$

$\mathrm{H}$

N

H

$\mathrm{H}$

O

$\mathrm{P}$

O

O

O

C

C $\quad-9.285$

C $\quad-9.977$

C $\quad-9.769$

O $\quad-9.425$

$\mathrm{H} \quad-9.867$

\begin{tabular}{|c|c|}
\hline-8.000 & 0.000 \\
\hline-9.254 & -1.873 \\
\hline-9.015 & -2.522 \\
\hline-8.671 & -2.624 \\
\hline-10.690 & -1.579 \\
\hline-9.530 & -1.790 \\
\hline-7.864 & 0.379 \\
\hline-8.954 & -1.526 \\
\hline-9.284 & -0.884 \\
\hline-8.435 & 0.199 \\
\hline-8.194 & -0.433 \\
\hline-12.417 & -0.102 \\
\hline-10.673 & 2.025 \\
\hline-11.143 & -0.254 \\
\hline-12.811 & 1.193 \\
\hline-11.951 & 2.267 \\
\hline-10.273 & 0.769 \\
\hline-8.640 & -2.220 \\
\hline-7.949 & 0.422 \\
\hline-8.518 & -1.721 \\
\hline-8.381 & -1.341 \\
\hline-8.036 & -0.022 \\
\hline-8.194 & -0.433 \\
\hline-6.955 & 1.391 \\
\hline-6.202 & 1.112 \\
\hline-6.586 & 1.850 \\
\hline-7.667 & 1.721 \\
\hline-5.425 & 1.398 \\
\hline-7.266 & 2.299 \\
\hline-9.808 & 3.038 \\
\hline-9.957 & 3.944 \\
\hline-8.838 & 2.758 \\
\hline-7.270 & 1.859 \\
\hline-7.197 & 2.204 \\
\hline-4.924 & -0.715 \\
\hline-4.897 & 0.871 \\
\hline-6.350 & -1.336 \\
\hline-3.457 & -1.540 \\
\hline-4.809 & -1.294 \\
\hline-3.849 & -1.065 \\
\hline-3.994 & -1.739 \\
\hline-3.076 & -1.552 \\
\hline-1.987 & -0.683 \\
\hline-1.817 & -0.009 \\
\hline-2.738 & -0.196 \\
\hline-1.025 & -0.476 \\
\hline-0.066 & 0.296 \\
\hline-1.208 & -1.078 \\
\hline-2.916 & -1.518 \\
\hline-3.487 & -0.264 \\
\hline-2.634 & 0.998 \\
\hline-4.798 & -0.135 \\
\hline-7.410 & 1.949 \\
\hline
\end{tabular}

\section{TS-4C}

C

C

C

C

C

C

C

N

C

C

C

C

$\mathrm{N}$

C

C

C

C

N

N

$\mathrm{H}$

$\mathrm{H}$

$\mathrm{H}$

$\mathrm{H}$

N

$\mathrm{H}$

$\mathrm{H}$

0

$\mathrm{H}$

P

0

0

0

0

C

C

C

C

C

C

N

O

C

C

C

8.000
7.973
9.291
6.847
7.709
11.672
11.204
10.430
12.701
12.482
10.185
8.793

8.472

8.282

9.173

9.006

8.151

4.327

4.839

5.620

3. 248

3.492

5.874

10.907

10.333

11.727

5.139

7.884

4.412

8.206

8.611

7.970

7.919

8.836

8.032

8.195

8.073

8.124

9.919

10.768

12.107

13.087

12.747

11.433

10.452

13.769

13.439

14.933

6.855

5.795

5.579

6.271

6.109
$-8.000$

0.000

$-9.226$

$-1.893$

$-9.013$

$-8.604$

$-10.656$

$-9.564$

$-7.864$

$-8.967$

$-9.328$

$-8.459$

$-8.193$

$-12.418$

$-10.671$

$-11.128$

$-12.833$

$-11.971$

$-10.255$

$-8.567$

$-7.969$

$-8.470$

$-8.331$

$-8.032$

$-8.193$

$-6.916$

$-6.152$

$-6.548$

$-7.734$

$-5.619$

$-7.334$

$-9.803$

$-9.979$

$-8.828$

$-7.255$

$-7.163$

$-4.844$

$-4.735$

$-6.281$

$-3.329$

$-4.780$

$-3.814$

$-3.929$

$-3.013$

$-1.950$

$-1.807$

$-2.728$

$-0.989$

$-0.044$

$-1.158$

$-2.704$

$-3.393$

$-2.705$

$-4.733$

$-7.484$
$-2.533$

$-2.638$

$-1.603$

$-1.752$

0.384

$-1.523$

$-0.819$

0.250

$-0.443$

$-0.128$

2.005

$-0.276$

1.166

2. 242

0.749

$-2.259$

0.408

$-1.745$

$-1.382$

$-0.049$

$-0.443$

1. 361

0.978

1.836

1. 721

1.470

2. 300

3. 023

3.934

2. 758

1.865

2. 191

$-0.573$

1.083

$-1.195$

$-1.317$

$-0.744$

$-0.372$

$-0.851$

$-0.484$

0.374

0.860

0.492

0.768

1.513

0.345

$-1.605$

$-0.718$

0.641

$-0.590$

1. 964 


\section{Supporting Information}

$3 d$

$\mathrm{N}$

c

c

c

C

C

C

C

C

C

C

$\mathrm{N}$

N

H

H

H

H

O

$\mathrm{H} \quad-7.566$

P $\quad-8.409$

O $\quad-9.458$

O $\quad-7.828$

O $\quad-7.770$

O $\quad-6.876$

C $\quad-6.547$

C $\quad-5.166$

C $\quad-4.756$

C $\quad-5.729$

C $\quad-7.101$

C $\quad-7.507$

$\mathrm{N} \quad-5.307$

O $\quad-6.189$

O $\quad-4.085$

C $\quad-8.421$

C $\quad-9.825$

C $\quad-10.905$

O $\quad-9.633$

$\mathrm{H} \quad-10.054$

$$
8.000
$$

6.895

7.102

7.557

5.460

6.473

8.043

7.081

6.631

7.426

7.797

3.656

5.395

4.956

3.225

4.082

5.819

7.489

7.880

7.604

7.602

7.795

7.797

8.908

9.666

9.221

7.994

10.193

8.295

6.269

6.071

7.249

8.724

9.291

11.128

10.951

9.811

12.672

11.086

12.097

12.382

13.372

14.115

13.876

12.880

15.154

15.815

15.330

13.445

12.836

13.447

11.439

8.299
0.000

$-1.959$

$-2.615$

$-2.688$

$-1.701$

$-1.907$

0.346

$-1.623$

$-0.975$

0.150

$-0.496$

$-0.278$

1.884

$-0.395$

0.995

2.082

0.645

$-2.372$

0.346

$-1.820$

$-1.506$

$-0.146$

$-0.496$

1.382

1.154

1.907

1.685

1.355

2. 274

2. 909

3.801

2.673

1.854

2.118

$-0.465$

0.800

$-1.061$

$-0.678$

0.894

1.711

1.922

2. 812

3.504

3.302

2.416

4.440

5.024

4.613

$-1.712$

$-1.910$

$-1.007$

$-1.650$

1.967
6

Zn

C

C

C

10.566

11.026

9.672

11.737

11.969

9.870

9.522

8.876

9.043

9.735

9.420

8.746

5.176

5.093

5.848

4.454

4.399

5.853

11.324

10.659

12.124

5.036

7.141

4.322

8.435

8.691

8.096

7.929

8.760

5.786

5.636

6.937

5.858

9.931

10.156

11.492

11.745

10.666

9.336

9.087

10.927

9.951

12.115

4.904

3.727

2.691

4.360

5.227
8.000

5.920

5.993

5.395

5.149

6.445

8.510

6.775

7.220

8.248

7.786

4.786

7.443

5.643

5.318

6.643

6.947

4.943

7.692

5.846

5.464

6.837

7.186

9.466

9.930

10.051

9.054

10.299

9.432

8.703

9.176

9.269

9.856

10.555

9.598

10.442

8.549

10.615

11.229

11.756

11.928

12.463

12.850

12.718

12.183

13.393

13.709

13.516

10.190

9.539

10.539

8.826

9.601

0.000

$-0.666$

$-2.086$

$-0.552$

0.152

$-3.237$

$-1.433$

$-2.220$

$-3.372$

$-2.475$

$-1.326$

2.576

3.075

1.585

3.865

4.123

1.805 
Supporting Information

1

Zn

C

C

C

C

C

C

$\mathrm{N}$

C

C

C

$\mathrm{N}$

O

$\mathrm{H}$

O

O

$-4.960$

C $\quad-4.376$

C $\quad-4.434$

C $\quad-5.086$

$\mathrm{N} \quad-3.688$

O -3.178

O $\quad-3.649$

C $\quad-9.048$

C $\quad-10.044$

C $\quad-9.578$

O -10.390

$\mathrm{H} \quad-9.569$

TS-2

$\begin{array}{rr}\text { Zn } & 8.000 \\ \text { N } & 8.421 \\ \text { C } & 9.847 \\ \text { C } & 7.511 \\ \text { C } & 8.187 \\ \text { C } & 11.999 \\ \text { C } & 10.792 \\ \text { C } & 10.683 \\ \text { C } & 12.728\end{array}$

$\begin{array}{rr} & \\ 8.000 & 0.000 \\ 6.818 & -2.019 \\ 7.010 & -2.268 \\ 7.541 & -3.001 \\ 5.396 & -1.878 \\ 6.322 & -1.039 \\ 7.281 & 1.270 \\ 6.838 & -1.006 \\ 6.304 & 0.146 \\ 6.796 & 1.323 \\ 7.298 & 0.133 \\ 3.562 & -0.099 \\ 5.505 & 1.771 \\ 4.925 & -0.432 \\ 3.178 & 1.225 \\ 4.167 & 2.179 \\ 5.869 & 0.491 \\ 7.662 & -3.292 \\ 8.299 & -0.620 \\ 7.734 & -2.464 \\ 7.927 & -2.742 \\ 8.254 & -1.384 \\ 8.038 & -1.148 \\ 8.618 & 1.810 \\ 9.176 & 2.126 \\ 10.777 & -0.306 \\ 10.495 & 0.503 \\ 9.672 & -1.122 \\ 11.592 & 0.677 \\ 11.920 & -1.576 \\ 13.101 & -1.415 \\ 13.919 & -2.566 \\ 15.150 & -2.517 \\ 15.562 & -1.307 \\ 14.759 & -0.157 \\ 13.524 & -0.206 \\ 16.858 & -1.248 \\ 17.201 & -0.166 \\ 17.546 & -2.284 \\ 11.924 & 0.234 \\ 11.732 & 1.395 \\ 12.423 & 2.691 \\ 105 & 1.615 \\ 1.793 & 1.762\end{array}$

$\begin{array}{lr}8.000 & 0.000 \\ 6.851 & -1.965 \\ 7.178 & -2.220 \\ 7.485 & -2.943 \\ 5.398 & -1.789 \\ 6.452 & -1.030 \\ 7.131 & 1.343 \\ 6.934 & -0.972 \\ 6.323 & 0.161\end{array}$

$\begin{array}{rrrr}\mathrm{C} & 12.113 & 6.665 & 1.372 \\ \mathrm{~N} & 10.092 & 7.264 & 0.199 \\ \mathrm{C} & 6.742 & 3.778 & -0.439 \\ \mathrm{C} & 6.706 & 5.609 & 1.611 \\ \mathrm{C} & 7.371 & 5.029 & -0.547 \\ \mathrm{C} & 6.084 & 3.448 & 0.754 \\ \mathrm{C} & 6.069 & 4.379 & 1.804 \\ \mathrm{~N} & 7.341 & 5.922 & 0.462 \\ \mathrm{C} & 4.968 & 7.589 & -3.207 \\ \mathrm{C} & 4.820 & 8.298 & -0.556 \\ \mathrm{C} & 6.106 & 7.669 & -2.389 \\ \mathrm{C} & 3.714 & 7.887 & -2.656 \\ \mathrm{C} & 3.637 & 8.254 & -1.305 \\ \mathrm{~N} & 6.024 & 8.005 & -1.084 \\ \mathrm{O} & 7.591 & 8.694 & 1.873 \\ \mathrm{H} & 7.032 & 9.649 & 1.460 \\ \mathrm{P} & 8.780 & 10.939 & -0.124 \\ \mathrm{O} & 9.375 & 10.733 & 1.288 \\ \mathrm{O} & 8.512 & 9.673 & -1.047 \\ \mathrm{O} & 8.077 & 12.410 & -0.500 \\ \mathrm{O} & 10.160 & 11.580 & -1.104 \\ \mathrm{C} & 11.389 & 11.054 & -1.140 \\ \mathrm{C} & 12.097 & 11.175 & -2.371 \\ \mathrm{C} & 13.380 & 10.654 & -2.515 \\ \mathrm{C} & 13.981 & 10.003 & -1.420 \\ \mathrm{C} & 13.319 & 9.912 & -0.181 \\ \mathrm{C} & 12.035 & 10.431 & -0.035 \\ \mathrm{~N} & 15.291 & 9.389 & -1.579 \\ \mathrm{O} & 15.756 & 8.727 & -0.624 \\ \mathrm{O} & 15.888 & 9.533 & -2.667 \\ \mathrm{C} & 6.707 & 12.715 & -0.177 \\ \mathrm{C} & 6.225 & 11.851 & 1.003 \\ \mathrm{C} & 6.694 & 12.357 & 2.380 \\ \mathrm{O} & 6.727 & 10.575 & 0.662 \\ \mathrm{H} & 8.416 & 9.200 & 2.078\end{array}$

$\begin{array}{crrr}\text { 3'a } & & & \\ \text { Zn } & -8.000 & -8.000 & 0.000 \\ \text { N } & -7.623 & -9.106 & -1.950 \\ \text { C } & -6.169 & -8.904 & -2.179 \\ \text { C } & -8.456 & -8.353 & -2.919 \\ \text { C } & -8.018 & -10.528 & -1.839 \\ \text { C } & -4.091 & -9.777 & -0.960 \\ \text { C } & -5.211 & -8.875 & 1.379 \\ \text { C } & -5.357 & -9.177 & -0.921 \\ \text { C } & -3.367 & -9.915 & 0.231 \\ \text { C } & -3.940 & -9.458 & 1.427 \\ \text { N } & -5.906 & -8.736 & 0.232 \\ \text { C } & -8.259 & -12.401 & -0.124 \\ \text { C } & -8.702 & -10.509 & 1.817 \\ \text { C } & -8.232 & -11.025 & -0.407 \\ \text { C } & -8.531 & -12.823 & 1.184 \\ \text { C } & -8.762 & -11.860 & 2.177 \\ \text { N } & -8.451 & -10.104 & 0.553 \\ \text { C } & -10.975 & -8.060 & -3.250 \\ \text { C } & -11.201 & -7.712 & -0.537\end{array}$


Supporting Information

$\begin{array}{lrrr}\mathrm{C} & -9.869 & -8.152 & -2.390 \\ \mathrm{C} & -12.236 & -7.771 & -2.709 \\ \mathrm{C} & -12.352 & -7.589 & -1.324 \\ \mathrm{~N} & -9.989 & -7.993 & -1.055 \\ \mathrm{O} & -8.265 & -7.279 & 1.946 \\ \mathrm{H} & -8.949 & -6.577 & 1.704 \\ \mathrm{P} & -7.361 & -5.050 & -0.003 \\ \mathrm{O} & -6.684 & -5.327 & 1.387 \\ \mathrm{O} & -7.562 & -6.304 & -0.983 \\ \mathrm{O} & -7.848 & -3.492 & -0.438 \\ \mathrm{O} & -5.853 & -4.490 & -0.961 \\ \mathrm{C} & -4.675 & -5.097 & -0.998 \\ \mathrm{C} & -3.995 & -5.126 & -2.256 \\ \mathrm{C} & -2.763 & -5.758 & -2.397 \\ \mathrm{C} & -2.171 & -6.370 & -1.273 \\ \mathrm{C} & -2.792 & -6.303 & -0.009 \\ \mathrm{C} & -4.025 & -5.677 & 0.134 \\ \mathrm{~N} & -0.933 & -7.109 & -1.425 \\ \mathrm{O} & -0.476 & -7.719 & -0.430 \\ \mathrm{O} & -0.381 & -7.130 & -2.547 \\ \mathrm{C} & -9.218 & -3.129 & -0.215 \\ \mathrm{C} & -9.771 & -4.015 & 0.915 \\ \mathrm{C} & -9.460 & -3.475 & 2.324 \\ \mathrm{O} & -9.168 & -5.274 & 0.659 \\ \mathrm{H} & -7.485 & -6.591 & 1.931\end{array}$

\begin{tabular}{|c|c|c|c|}
\hline \multicolumn{4}{|c|}{ TS-4 ' } \\
\hline $\mathrm{Zn}$ & 8.000 & -8.000 & 0.000 \\
\hline $\mathrm{N}$ & 8.494 & -9.049 & -1.951 \\
\hline $\mathrm{C}$ & 9.907 & -8.652 & -2.189 \\
\hline $\mathrm{C}$ & 7.572 & -8.422 & -2.925 \\
\hline $\mathrm{C}$ & 8.318 & -10.513 & -1.813 \\
\hline $\mathrm{C}$ & 12.118 & -9.318 & -1.098 \\
\hline $\mathrm{C}$ & 10.900 & -9.041 & 1.338 \\
\hline $\mathrm{C}$ & 10.768 & -8.954 & -0.974 \\
\hline $\mathrm{C}$ & 12.877 & -9.530 & 0.059 \\
\hline $\mathrm{C}$ & 12.251 & -9.401 & 1.307 \\
\hline $\mathrm{N}$ & 10.167 & -8.809 & 0.229 \\
\hline C & 6.998 & -12.250 & -0.500 \\
\hline $\mathrm{C}$ & 6.777 & -10.471 & 1.577 \\
\hline $\mathrm{C}$ & 7.526 & -10.951 & -0.577 \\
\hline $\mathrm{C}$ & 6.347 & -12.658 & 0.671 \\
\hline $\mathrm{C}$ & 6.237 & -11.751 & 1.737 \\
\hline $\mathrm{N}$ & 7.410 & -10.082 & 0.448 \\
\hline $\mathrm{C}$ & 5.031 & -8.390 & -3.238 \\
\hline $\mathrm{C}$ & 4.808 & -7.831 & -0.557 \\
\hline $\mathrm{C}$ & 6.150 & -8.319 & -2.392 \\
\hline $\mathrm{C}$ & 3.757 & -8.165 & -2.698 \\
\hline C & 3.641 & -7.876 & -1.331 \\
\hline $\mathrm{N}$ & 6.032 & -8.056 & -1.074 \\
\hline 0 & 7.794 & -7.420 & 2.067 \\
\hline $\mathrm{H}$ & 7.687 & -6.427 & 1.899 \\
\hline $\mathrm{P}$ & 8.389 & -5.001 & 0.013 \\
\hline 0 & 8.889 & -5.021 & 1.492 \\
\hline 0 & 8.568 & -6.336 & -0.881 \\
\hline 0 & 7.705 & -3.609 & -0.676 \\
\hline
\end{tabular}

$\begin{array}{rrrr}\text { O } & 9.910 & -4.250 & -0.809 \\ \mathrm{C} & 11.138 & -4.732 & -0.717 \\ \mathrm{C} & 12.005 & -4.524 & -1.836 \\ \mathrm{C} & 13.300 & -5.030 & -1.851 \\ \mathrm{C} & 13.774 & -5.749 & -0.731 \\ \mathrm{C} & 12.961 & -5.911 & 0.410 \\ \mathrm{C} & 11.663 & -5.414 & 0.424 \\ \mathrm{~N} & 15.086 & -6.356 & -0.769 \\ \mathrm{O} & 15.441 & -7.075 & 0.198 \\ \mathrm{O} & 15.809 & -6.164 & -1.772 \\ \mathrm{C} & 6.279 & -3.486 & -0.599 \\ \mathrm{C} & 5.779 & -4.396 & 0.543 \\ \mathrm{C} & 5.808 & -3.718 & 1.925 \\ \mathrm{O} & 6.635 & -5.526 & 0.463 \\ \mathrm{H} & 8.679 & -7.421 & 2.475\end{array}$

\begin{tabular}{|c|c|c|c|}
\hline \multicolumn{4}{|c|}{3 'b } \\
\hline $\mathrm{Zn}$ & -8.000 & 8.000 & 0.000 \\
\hline $\mathrm{N}$ & -7.603 & 6.763 & -1.967 \\
\hline $\mathrm{C}$ & -6.187 & 7.088 & -2.246 \\
\hline C & -8.533 & 7.358 & -2.955 \\
\hline C & -7.818 & 5.316 & -1.738 \\
\hline C & -3.991 & 6.552 & -1.047 \\
\hline C & -5.200 & 7.266 & 1.309 \\
\hline C & -5.335 & 6.952 & -0.992 \\
\hline C & -3.236 & 6.526 & 0.134 \\
\hline C & -3.852 & 6.891 & 1.339 \\
\hline N & -5.926 & 7.295 & 0.175 \\
\hline C & -9.454 & 3.806 & -0.477 \\
\hline C & -9.462 & 5.649 & 1.565 \\
\hline C & -8.721 & 5.001 & -0.546 \\
\hline C & -10.200 & 3.533 & 0.679 \\
\hline C & -10.204 & 4.470 & 1.724 \\
\hline N & -8.729 & 5.899 & 0.460 \\
\hline C & -11.083 & 7.521 & -3.152 \\
\hline C & -11.125 & 8.380 & -0.539 \\
\hline C & -9.917 & 7.614 & -2.377 \\
\hline C & -12.309 & 7.889 & -2.579 \\
\hline C & $-12 \cdot 332$ & 8.331 & -1.249 \\
\hline $\mathrm{N}$ & -9.949 & 8.028 & -1.094 \\
\hline 0 & -8.772 & 8.782 & 1.634 \\
\hline $\mathrm{H}$ & -8.981 & 10.206 & 0.978 \\
\hline $\mathrm{P}$ & -7.775 & 10.975 & -0.689 \\
\hline 0 & -9.072 & 11.010 & 0.325 \\
\hline 0 & -7.358 & 9.552 & -1.225 \\
\hline 0 & -6.893 & 12.419 & -0.819 \\
\hline O & -6.574 & 10.687 & 0.774 \\
\hline C & -6.369 & 11.461 & 1.840 \\
\hline C & -5.262 & 11.126 & 2.676 \\
\hline C & -4.983 & 11.842 & 3.835 \\
\hline C & -5.811 & 12.925 & 4.189 \\
\hline C & -6.906 & 13.287 & 3.381 \\
\hline C & -7.184 & 12.569 & 2.221 \\
\hline N & -5.529 & 13.677 & 5.403 \\
\hline 0 & -6.271 & 14.639 & 5.690 \\
\hline 0 & -4.559 & 13.324 & 6.107 \\
\hline
\end{tabular}


Supporting Information

\begin{tabular}{|c|c|c|c|c|c|c|c|}
\hline C & -7.240 & 13.248 & -1.946 & C & -7.526 & -9.300 & -2.684 \\
\hline C & -8.648 & 12.815 & -2.408 & C & -9.864 & -9.452 & -1.897 \\
\hline C & -9.791 & 13.594 & -1.738 & C & -8.171 & -10.990 & -0.986 \\
\hline 0 & -8.694 & 11.419 & -2.112 & $\mathrm{C}$ & -4.987 & -9.168 & -2.948 \\
\hline \multirow[t]{3}{*}{$\mathrm{H}$} & -8.106 & 9.005 & 2.304 & C & -4.904 & -7.706 & -0.612 \\
\hline & & & & C & -6.139 & -8.893 & -2.201 \\
\hline & & & & $\mathrm{C}$ & -3.753 & -8.668 & -2.502 \\
\hline \multicolumn{2}{|c|}{ TS-5' } & & & C & -3.713 & -7.924 & -1.318 \\
\hline $\mathrm{Zn}$ & 8.000 & 8.000 & 0.000 & $\mathrm{~N}$ & -6.092 & -8.181 & -1.053 \\
\hline $\mathrm{N}$ & 8.394 & 6.823 & -1.932 & C & -6.639 & -12.141 & 0.690 \\
\hline C & 9.809 & 7.172 & -2.209 & C & -6.665 & -9.852 & 2.207 \\
\hline $\mathrm{C}$ & 7.463 & 7.418 & -2.919 & $\mathrm{C}$ & -7.321 & -10.984 & 0.282 \\
\hline C & 8.199 & 5.372 & -1.718 & C & -5.957 & -12.134 & 1.916 \\
\hline C & 12.013 & 6.623 & -1.034 & C & -5.975 & -10.968 & 2.695 \\
\hline C & 10.797 & 7.247 & 1.347 & $\mathrm{~N}$ & -7.324 & -9.858 & 1.028 \\
\hline C & 10.665 & 7.002 & -0.963 & C & -12.024 & -9.693 & -0.530 \\
\hline C & 12.769 & 6.566 & 0.146 & $\mathrm{C}$ & $-10 \cdot 792$ & -8.259 & 1.471 \\
\hline C & 12.150 & 6.884 & 1.363 & $\mathrm{C}$ & -10.696 & -9.251 & -0.637 \\
\hline $\mathrm{N}$ & 10.069 & 7.300 & 0.215 & $\mathrm{C}$ & $-12 \cdot 749$ & -9.381 & 0.630 \\
\hline C & 6.633 & 3.803 & -0.452 & C & $-12 \cdot 126$ & -8.648 & 1.649 \\
\hline $\mathrm{C}$ & 6.542 & 5.638 & 1.589 & $\mathrm{~N}$ & -10.097 & -8.558 & 0.356 \\
\hline C & 7.312 & 5.030 & -0.522 & 0 & -7.819 & -6.744 & 1.554 \\
\hline C & 5.899 & 3.496 & 0.702 & $\mathrm{H}$ & -8.193 & -5.878 & 1.211 \\
\hline C & 5.854 & 4.429 & 1.748 & $\mathrm{P}$ & -9.077 & -5.241 & -1.245 \\
\hline $\mathrm{N}$ & 7.264 & 5.927 & 0.485 & 0 & -8.982 & -4.761 & 0.207 \\
\hline C & 4.919 & 7.530 & -3.154 & 0 & -8.660 & -6.720 & -1.543 \\
\hline C & 4.809 & 8.375 & -0.541 & 0 & -8.262 & -4.262 & -2.356 \\
\hline C & 6.066 & 7.637 & -2.354 & 0 & -5.414 & -6.519 & 2.236 \\
\hline C & 3.675 & 7.875 & -2.607 & $\mathrm{C}$ & -4.955 & -6.853 & 3.412 \\
\hline C & 3.618 & 8.313 & -1.276 & $\mathrm{C}$ & -3.550 & -7.083 & 3.612 \\
\hline $\mathrm{N}$ & 6.004 & 8.039 & -1.066 & C & -3.047 & -7.500 & 4.838 \\
\hline 0 & 7.205 & 8.756 & 1.744 & $\mathrm{C}$ & -3.927 & -7.699 & 5.924 \\
\hline $\mathrm{H}$ & 7.011 & 9.772 & 1.306 & $\mathrm{C}$ & -5.309 & -7.451 & 5.782 \\
\hline $\mathrm{P}$ & 8.105 & 10.975 & -0.433 & C & -5.813 & -7.032 & 4.554 \\
\hline 0 & 6.907 & 10.917 & 0.598 & $\mathrm{~N}$ & -3.408 & -8.161 & 7.195 \\
\hline 0 & 8.558 & 9.609 & -1.094 & 0 & -4.210 & -8.340 & 8.139 \\
\hline 0 & 9.159 & 12.292 & -0.335 & 0 & -2.177 & -8.369 & 7.296 \\
\hline 0 & 9.454 & 10.352 & 1.146 & C & -9.202 & -3.307 & -2.895 \\
\hline C & 9.757 & 11.022 & 2.237 & C & -10.540 & -4.060 & -3.039 \\
\hline C & 10.956 & 10.686 & 2.952 & $\mathrm{C}$ & -11.770 & -3.157 & -3.074 \\
\hline C & 11.306 & 11.319 & 4.139 & 0 & $-10 \cdot 618$ & -4.930 & -1.875 \\
\hline C & 10.469 & 12.328 & 4.658 & $\mathrm{H}$ & -6.833 & -6.580 & 1.906 \\
\hline
\end{tabular}

All hydrogen atoms on either alkyl or aryl carbon atoms are not included in this list. 
Electronic energies (E), zero-point energies (ZPE), enthalpies (H), free energies (G) (in Hartree) and solvation free energies $\left(\Delta \mathrm{G}_{\text {solv }}\right)$ (in $\mathrm{kcal} / \mathrm{mol}$ ) for all species included in Figure 1 and 2

\begin{tabular}{lccccc}
\hline & $\mathrm{E}$ & $\mathrm{ZPE}$ & $\mathrm{H}$ & $\mathrm{G}$ & $\Delta \mathrm{G}_{\text {solv }}$ \\
\hline 1 & -2322.66280505 & -2322.066139 & -2322.020810 & -2322.146760 & -5.18 \\
TS-2 & -2322.63672673 & -2322.042717 & -2321.999398 & -2322.118379 & -9.17 \\
3a & -2322.64227440 & -2322.044669 & -2322.000973 & -2322.120691 & -12.80 \\
TS-4a & -2322.63258107 & -2322.034671 & -2321.991270 & -2322.110579 & -13.16 \\
3b & -2322.63355707 & -2322.035921 & -2321.991864 & -2322.113141 & -12.46 \\
TS-4b & -2322.63333930 & -2322.038154 & -2321.994544 & -2322.114813 & -11.61 \\
3c & -2322.64050391 & -2322.042740 & -2321.998010 & -2322.121851 & -7.16 \\
TS-4c & -2322.63831437 & -2322.040935 & -2321.997138 & -2322.117086 & -9.17 \\
3d & -2322.64515585 & -2322.047995 & -2322.003273 & -2322.126379 & -8.50 \\
6 & -2322.67220639 & -2322.075901 & -2322.030867 & -2322.157619 & -10.13 \\
1' & -2156.49984769 & -2155.952251 & -2155.910303 & -2156.030457 & -10.44 \\
TS-2' & -2156.47657656 & -2155.932237 & -2155.891596 & -2156.006745 & -8.85 \\
3'a & -2156.47948270 & -2155.931926 & -2155.890924 & -2156.007079 & -10.96 \\
TS-4' & -2156.46638753 & -2155.919260 & -2155.878260 & -2155.994286 & -15.13 \\
3'b & -2156.48516999 & -2155.937783 & -2155.896715 & -2156.013143 & -10.49 \\
TS-5' & -2156.48088933 & -2155.935416 & -2155.894828 & -2156.011164 & -14.49 \\
6' & -2156.52285045 & -2155.975379 & -2155.933817 & -2156.055253 & -12.51 \\
\hline
\end{tabular}

